OPEN ACCESS

Edited by:

Ruben Pio,

University of Navarra, Spain

Reviewed by:

Marcin Okrój

University of Gdańsk, Poland

Daniel Ajona,

University of Navarra, Spain

${ }^{*}$ Correspondence:

Chiara Agostinis

cagostinis@units.it

Specialty section: This article was submitted to Cancer Immunity and Immunotherapy,

a section of the journal

Frontiers in Immunology

Received: 10 December 2018 Accepted: 04 April 2019

Published: 03 May 2019

Citation:

Mangogna A, Agostinis C, Bonazza D, Belmonte B, Zacchi P, Zito G,

Romano A, Zanconati F, Ricci G, Kishore $U$ and Bulla $R$ (2019) Is the Complement Protein C19 a Pro- or Anti-tumorigenic Factor?

Bioinformatics Analysis Involving

Human Carcinomas.

Front. Immunol. 10:865.

doi: 10.3389/fimmu.2019.00865

\section{Is the Complement Protein C1q a Pro- or Anti-tumorigenic Factor? Bioinformatics Analysis Involving Human Carcinomas}

\author{
Alessandro Mangogna ${ }^{1}$, Chiara Agostinis ${ }^{2 *}$, Deborah Bonazza ${ }^{3}$, Beatrice Belmonte ${ }^{4}$, \\ Paola Zacchi ${ }^{1}$, Gabriella Zito ${ }^{2}$, Andrea Romano ${ }^{3}$, Fabrizio Zanconati ${ }^{3}$, Giuseppe Ricci ${ }^{2,3}$, \\ Uday Kishore ${ }^{5}$ and Roberta Bulla ${ }^{1}$

\footnotetext{
${ }^{1}$ Department of Life Sciences, University of Trieste, Trieste, Italy, ${ }^{2}$ Institute for Maternal and Child Health, Istituto di Ricovero e Cura a Carattere Scientifico (IRCCS) Burlo Garofolo, Trieste, Italy, ${ }^{3}$ Department of Medical, Surgical and Health Science, University of Trieste, Trieste, Italy, ${ }^{4}$ Tumor Immunology Unit, Human Pathology Section, Department of Health Sciences, University of Palermo, Palermo, Italy, ${ }^{5}$ Biosciences, College of Health and Life Sciences, Brunel University London, Uxbridge, United Kingdom
}

$\mathrm{C} 1 \mathrm{q}$ is the first subcomponent of the classical pathway of the complement system and belongs to the C1q/Tumor Necrosis Factor superfamily. C1q can perform a diverse range of immune and non-immune functions in a complement-dependent as well as -independent manner. Being a pattern recognition molecule of the innate immunity, C1q can recognize a number of self, non-self and altered-self ligands and bring about effector mechanisms designed to clear pathogens via opsonisation and inflammatory response. C1q is locally synthesized by macrophages and dendritic cells, and thus, can get involved in a range of biological processes, such as angiogenesis and tissue remodeling, immune modulation, and immunologic tolerance. The notion of $\mathrm{C} 1 \mathrm{q}$ involvement in the pathogenesis of cancer is still evolving. C1q appears to have a dual role in cancer: tumor promoting as well as tumor-protective, depending on the context of the disease. In the current study, we performed a bioinformatics analysis to investigate whether $\mathrm{C} 1 \mathrm{q}$ can serve as a potential prognostic marker for human carcinoma. We used the Oncomine database and the survival analysis platforms Kaplan-Meier plotter. Our results showed that high levels of $\mathrm{C} 1 \mathrm{q}$ have a favorable prognostic index in basal-like breast cancer for disease-free survival, and in HER2-positive breast cancer for overall survival, while it showed a pro-tumorigenic role of $\mathrm{C} 1 \mathrm{q}$ in lung adenocarcinoma, and in clear cell renal cell carcinoma. This in silico study, if validated via a retrospective study, can be a step forward in establishing $\mathrm{C} 1 \mathrm{q}$ as a new tool as a prognostic biomarker for various carcinoma.

\footnotetext{
Keywords: complement, classical pathway, C1q, tumor, microenvironment, prognosis
} 


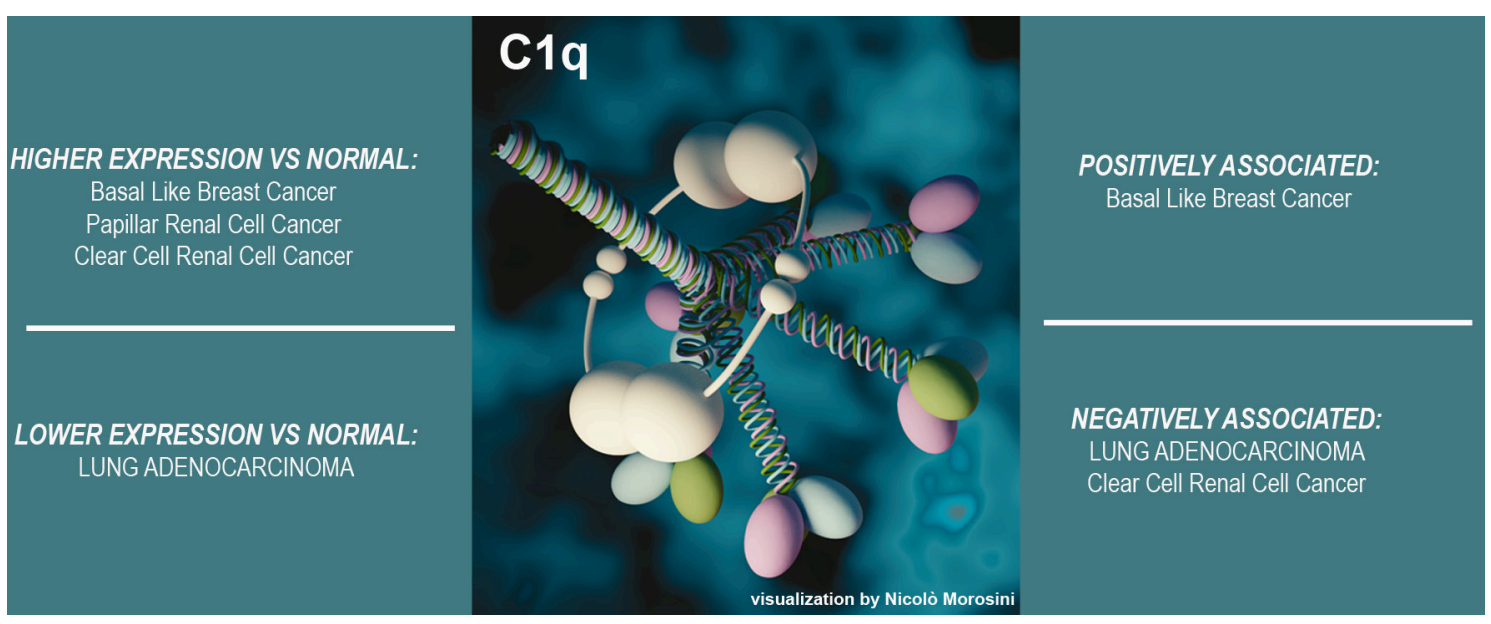

GRAPHICAL ABSTRACT | Summary of the conclusions of the study.

\section{INTRODUCTION}

$\mathrm{Clq}$ is the first recognition subcomponent of the complement classical pathway, which when associated with $\mathrm{C} 1 \mathrm{r}$ and $\mathrm{C} 1 \mathrm{~s}$, forms a $\mathrm{C} 1$ complex, allowing the activation of the complement cascade (1). By virtue of its ability to bind to IgG and IgM containing immune complexes and activating the classical pathway, $\mathrm{C1q}$ acts as prototypical link between innate and adaptive immune wings of the immune system (2). C1q can bind to a range of non-self-target ligands (pathogens), altered self ( $\beta$-amyloid peptide, prion protein, apoptotic and necrotic cells via phosphatidylserine and DNA, respectively), and cell surface receptors (such as calreticulin and gC1qR) (3). Several features of the $\mathrm{Clq}$ render it a versatile molecular sensor of damage-modified self or non-self antigens (4). C1q, unlike most of the complement proteins which are exclusively produced by hepatocytes, can also be synthesized in a local environment by a wide range of cell types including macrophages and dendritic cells (5). Local synthesis, therefore, offers an additional avenue to $\mathrm{C} 1 \mathrm{q}$ in order to exert specific functions in situ that are strictly connected to its site of production without involving complement activation (6).

$\mathrm{C} 1 \mathrm{q}$ is an hexametric glycoprotein of about $460 \mathrm{kDa}$, resembling a "bouquet of tulips" being composed by three polypeptide chains: A $(28 \mathrm{kDa}), \mathrm{B}(25 \mathrm{kDa}), \mathrm{C}(24 \mathrm{kDa})$, which are the product of three distinct genes clustered in the same orientation, and in the order $\mathrm{A}-\mathrm{C}-\mathrm{B}$, on a $24 \mathrm{~kb}$ stretch of DNA on chromosome 1p (7). Each chain consists of a C-terminal globular head (gC1q) domain and an N-terminal triple-helical collagen-like (cC1q) domain (8). C1q associates with the $\mathrm{Ca}^{2+}$. dependent $\mathrm{C}_{1} \mathrm{r}_{2}-\mathrm{C} 1 \mathrm{~s}_{2}$ tetramer, of about $360 \mathrm{kDa}$, to form the soluble pentameric C1 complex (9). The C-terminal ends of A, $\mathrm{B}$ and $\mathrm{C}$ chains assemble together to form a heterotrimeric $\mathrm{gClq}$

Abbreviations: TME, Tumor microenvironment; ECM, extracellular matrix; BLBC, basal-like breast cancer; CCRCC, clear cell renal cell carcinoma; PRCC, papillary renal cell carcinoma; OS, overall survival; DFS, disease-free survival; WOX1, WW-domain containing oxidoreductase. domain, which by virtue of its modular organization, can work independently and engage with a diverse range of target ligands (3). While the gC1q domain latches on to the charge patterns on the ligands, the $\mathrm{cClq}$ domain can interact with effector mechanism inducers, such as $\mathrm{C} 1 \mathrm{r}, \mathrm{C} 1 \mathrm{~s}$, cell surface receptors, etc. Thus, a combination of a highly versatile and modular gC1q domain and a cell surface interacting cC1q domain, together with its local synthesis, makes $\mathrm{C} 1 \mathrm{q}$ a potent orchestrator of molecular pathways. $\mathrm{C} 1 \mathrm{q}$ is involved not only in innate and adaptive immune mechanisms, but also in a wide range of physiological and pathological processes, such as placental development $(10,11)$, pre-eclampsia $(12,13)$, wound healing (14) and cancer (15-18).

Markiewski et al. provided evidence that $\mathrm{Clq}$ is present in syngeneic mouse tumors. Indeed, they found that the activation of the classical pathway is the major contributor to complement-mediated tumor progression (19). Subsequently, we showed that locally expressed $\mathrm{Clq}$ had important effects in the tumor microenvironment (TME) (17). C1q expressed in the stroma and vascular endothelium of several human malignant tumors acted as a tumor-promoting factor by favoring adhesion, migration and proliferation of cancer cells as well as angiogenesis and metastasis. C1q-deficient $\left(\mathrm{Clqa}^{-/}\right)$mice, bearing a syngeneic B16 melanoma, exhibited slower tumor growth and prolonged survival, compared to C3 or C5 deficient mice although it has been shown that C3/C5 deficiency may also create microenvironment suboptimal for tumor growth $(20,21)$. Recently, we demonstrated that $\mathrm{Clq}$ is abundantly present in malignant pleural mesothelioma (MPM), where it can combine with hyaluronic acid (HA), which is a principal component of the TME, and enhance the tumor growth by promoting cell adhesion and proliferation (18). However, other have shown a pro-apoptotic effect of $\mathrm{Clq}$ on prostate (15) and ovarian cancer cells in vitro (16). These rather two set of contradicting studies warranted a systematic analysis of the context of the disease and TME that can render $\mathrm{Clq}$ protective or pathogenic in cancer.

In the current study, we performed a bioinformatics analysis, using Oncomine database and the survival analysis platforms 
Kaplan-Meier plotter, in order to investigate whether $\mathrm{C} 1 \mathrm{q}$ can serve as a potential prognostic marker for human carcinoma, i.e., tumors of epithelial origin. Our results showed that high levels of C1q have a favorable prognostic index in basal-like breast cancer (BLBC) and in HER-2 positive breast cancer. However, we found a pro-tumorigenic role of $\mathrm{Clq}$ in lung adenocarcinoma, and in clear cell renal cell carcinoma (CCRCC). This study is an important step forward in highlighting $\mathrm{Clq}$ as a new prognostic candidate biomarker for a range of carcinomas.

\section{METHODS}

\section{Oncomine Database Analysis}

The expression levels of $C 1 Q A, C 1 Q B$, and $C 1 Q C$ genes in various carcinomas were analyzed using Oncomine (www.oncomine. org), a cancer microarray database and web-based data mining platform from genome-wide expression analyses $(22,23)$. We compared the differences in mRNA level between normal tissue and carcinoma. The mRNA expression levels in neoplastic tissues compared to the healthy tissues were obtained as the parameters of $p$-value $<0.05$, fold change $>2$, and gene ranking in the top $10 \%$. Information about the dataset used in this study is summarized in Supplementary Table 1.

\section{Kaplan-Meier Plotter Database Analysis}

A Kaplan-Meier plotter database can be used to assess the effect of 54,675 genes on survival using 10,461 carcinoma samples $(5,143$ breast, 1,816 ovarian, 2,437 lung, and 1,065 gastric cancer patients with a mean follow-up of 69/40/49/33 months) using probe sets on the HGU133 Plus 2.0 array from Gene Expression Omnibus (GEO). For other human carcinoma, a total of 3,439 patients with RNA HiSeq data from The Cancer Genome Atlas (TCGA) cohort were collected. The prognostic significance of $C 1 Q A, C 1 Q B$, and $C 1 Q C$ expression and survival in several carcinomas was analyzed by Kaplan-Meier plotter (www.kmplot.com/analysis/) (24). The hazard ratio with $95 \%$ confidence intervals and logrank $p$-value was also computed.

\section{Immunohistochemical Analysis}

Normal and neoplastic human tissues, including breast, kidney and lung, were selected from the archives of the Department of Pathology, University of Trieste. Immunohistochemistry (IHC) was performed using a polymer detection method. Briefly, tissue samples were fixed in $10 \% \mathrm{v} / \mathrm{v}$ buffered formalin and then paraffin embedded. Four $\mu \mathrm{m}$-thick tissue sections were deparaffinized and rehydrated. The antigen unmasking technique was carried out using Novocastra Epitope Retrieval Solutions, pH 9 (Leica Biosystems) in a PT Link pre-treatment module (Dako) at $98^{\circ} \mathrm{C}$ for $30 \mathrm{~min}$. Sections were then brought to RT and washed in PBS. After neutralization of the endogenous peroxidase with $3 \% \mathrm{v} / \mathrm{v} \mathrm{H}_{2} \mathrm{O}_{2}$ and $\mathrm{Fc}$ blocking by a specific protein block (Novocastra, Leica Biosystems), samples were incubated overnight at $4^{\circ} \mathrm{C}$ with rabbit polyclonal anti-human C1q (dilution 1:200) antibodies (Dako). Staining was carried out via polymer detection kit (Novocastra, Leica Biosystems) and DAB (3,3'-Diaminobenzidine; Dako, Denmark) substrate-chromogen. Slides were counterstained with Harris
Haematoxylin (Novocastra, Leica Biosystems). Sections were analyzed under the Axio Scope A1 optical microscope (Zeiss) and microphotographs were collected through the Axiocam 503 color digital camera (Zeiss) using the Zen2 software.

\section{Statistical Analysis}

Survival curves were generated by the Kaplan-Meier plotter. All results are displayed with $p$-values from a log-rank test. $P$-values $<0.05$ were considered significant. Similarly, with Oncomine, the statistical significance of data ( $p$-values) was provided by the program.

\section{RESULTS}

\section{Bioinformatic Analysis of the Three Genes Encoding Human C1q A, B, and C Chains in Normal Epithelial Tissues and Carcinomas}

The expression of $C 1 Q A, C 1 Q B$, and $C 1 Q C$ genes was analyzed between different carcinoma and normal tissue counterparts using the Oncomine database. The threshold was determined as the following values: $p$-value $<0.05$, fold change $>2$, and gene ranking in the top $10 \%$. Carcinomas included in this analysis were: bladder carcinoma, breast cancer, cervical squamous cell carcinoma, esophageal carcinoma, head-neck squamous cell carcinoma, clear cell renal cell carcinoma (CCRCC), papillary renal cell carcinoma (PRCC), liver hepatocellular carcinoma, lung adenocarcinoma, lung squamous cell carcinoma, ovarian cancer, pancreatic ductal adenocarcinoma, rectum adenocarcinoma, gastric carcinoma, and uterine corpus endometrial carcinoma. We only investigated carcinomas in which all the three $\mathrm{Clq}$ chains showed a significant prognostic effect by Kaplan-Meier plotter analysis. The C1QA, $C 1 Q B$, and $C 1 Q C$ genes were either overexpressed, or downregulated depending on the type of carcinoma investigated, as compared to their normal tissue counterparts. All the three $\mathrm{Clq}$ chains showed a differential prognostic significance. These data appear to suggest that $\mathrm{Clq}$ can have proor anti-tumorigenic implications, depending on the carcinoma types (Table 1). Thus, detailed analyses of the expression profiles of all three $\mathrm{Clq}$ chains were performed.

\section{Significance of C1q Expression in Breast Carcinoma}

Bioinformatics analysis of $C 1 Q A, C 1 Q B$, and $C 1 Q C$ mRNA expression was performed in the context of the breast cancer using Karnoub's, Finak's, Curtis's, and Perou's datasets. A higher expression level of the three chains of $\mathrm{C} 1 \mathrm{q}$ was detected as compared to normal breast tissue (Figure 1A, $p<0.05$ ). When breast cancer was stratified into different histological subtypes, $C 1 Q A, C 1 Q B$, and $C 1 Q C$ mRNA expression achieved a statistical significance only in medullary carcinoma (Figure 1B, $p<0.05$ ). To evaluate the prognostic significance of $\mathrm{Clq}$ in all breast cancers, we considered their molecular classification, such as 
TABLE 1 | Prognostic significance of C1q in patients with carcinomas.

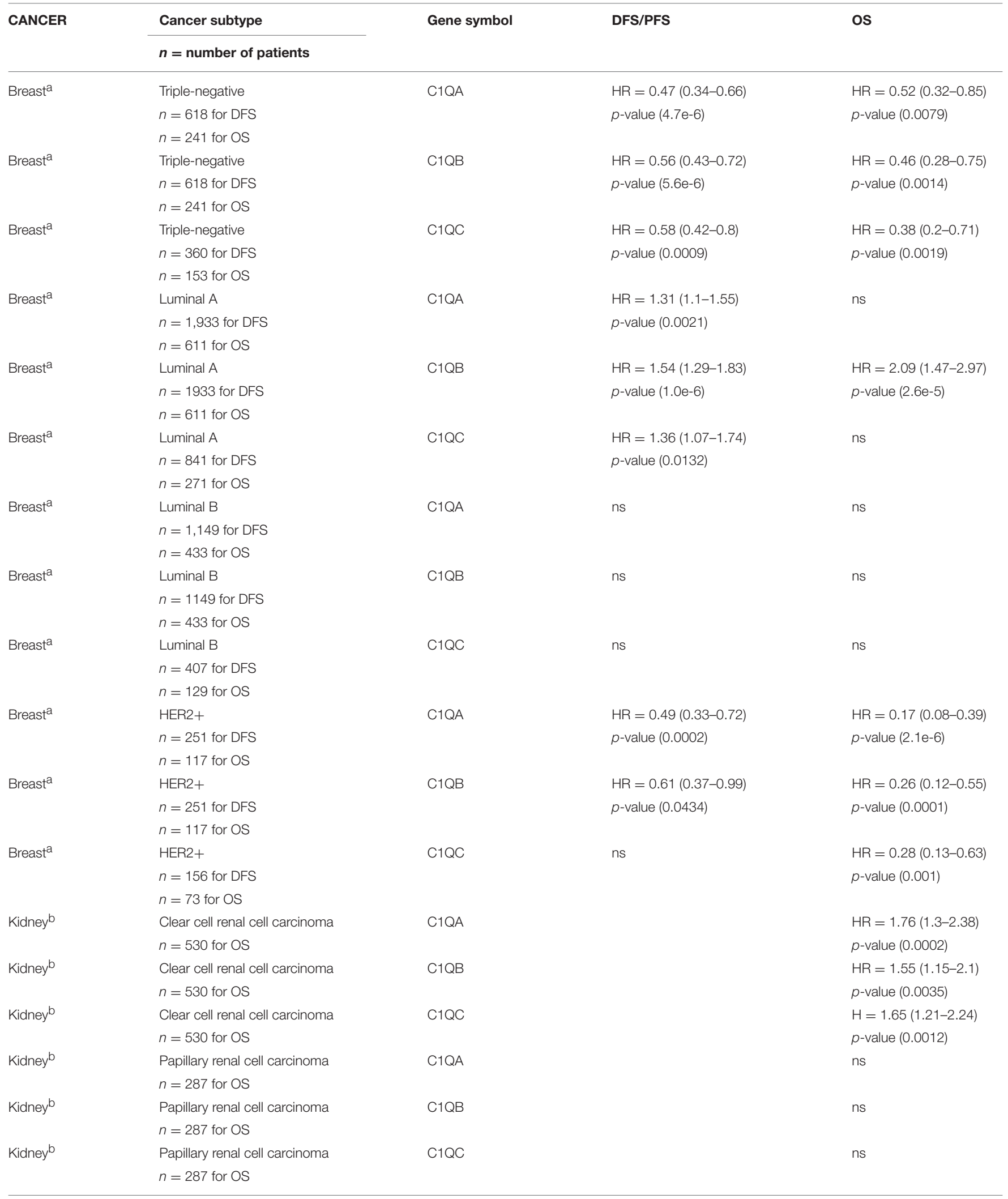


TABLE 1 | Continued

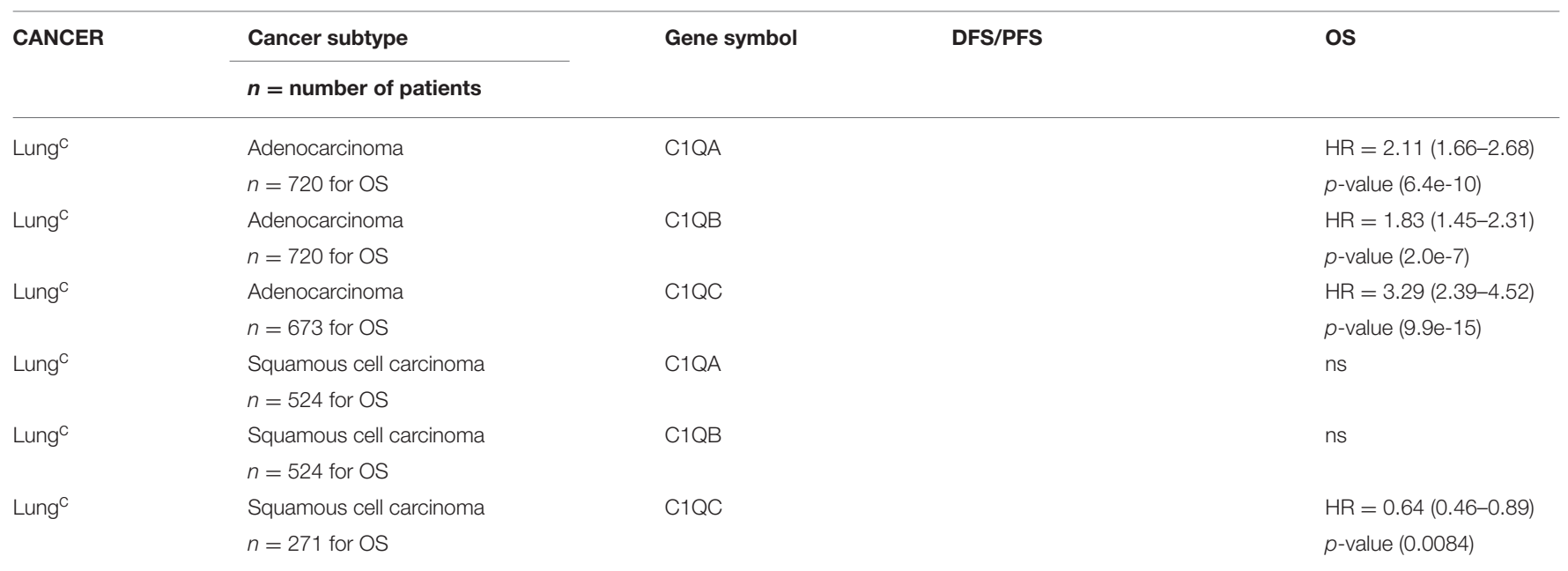

a Using 5,143 cancer samples on the HGU133 Plus 2.0 array from Gene Expression Omnibus, GEO.

bUsing 817 cancer samples on the RNA HiSeq data from The Cancer Genome Atlas, TCGA.

'Using 2,437 cancer samples on the HGU133 Plus 2.0 array from GEO.

DSF, disease-free survival; OS, overall survival.

luminal-A, luminal-B, HER-2 positive, and basal-like cancers (BLBC) (Supplementary Table 2).

According to Kaplan-Meir plotter data, $C 1 Q A, C 1 Q B$, and $C 1 Q C$ mRNA expression was positively associated with a diseasefree survival (DFS) rate in patients with BLBC (Figure 1C, $p<0.05$ ) and with an overall survival (OS) rate with HER-2 positive cancers (Table 1). This correlation was not evident in luminal-A and luminal-B patients. Only $C 1 Q B$ mRNA expression was negatively associated with high DFS and OS rates in the breast cancer patients with luminal-A, and to a DFS rate with all breast cancer.

The IHC analysis within the BLBC microenvironment revealed that $\mathrm{C} 1 \mathrm{q}$ was diffusely present in the tumor stroma and was expressed by macrophage-like cells, suggestive of tumorinfiltrating myeloid elements (Figure 2A).

\section{C1q Expression in Kidney Carcinoma has a Negative Correlation}

The $C 1 Q A, C 1 Q B$, and $C 1 Q C$ mRNA expression was evaluated in kidney cancer based on the results obtained from different datasets. In CCRCC, the expression of the three C1q chains was higher as compared to normal kidney (Figure 3A, $p<0.05$ ). However, in the case of PRCC, this trend was evident only for $C 1 Q A$ and $C 1 Q B$ mRNA expression (data not shown). The data obtained from Kaplan-Meier plotter showed a negative relationship between $C 1 Q A, C 1 Q B$, and $C 1 Q C$ mRNA expression and OS rate of patients with CCRCC (Figure 3B, $p<0.05$ ). No correlation was observed between $C 1 Q A, C 1 Q B$, and $C 1 Q C$ mRNA expression and OS in the PRCC patients (Table 1).

Within the CCRCC microenvironment, C1q was found to be mainly expressed in the tumor stroma and in the small vessels, and it was associated with the cell membrane of tumor cells (Figure 2B).

\section{Lower Level of C1q Expression in Lung Carcinoma}

While examing $C 1 Q A$ and $C 1 Q B$ mRNA expression in lung cancer, using Selamat's, Wachi's and Bhattacharjee's datasets, we found a lower expression level in adenocarcinoma (Figure 3C, $p<0.05$ ) and in squamous cell carcinoma (data not shown, $p<0.05$ ) than in normal lung tissue; C1QC mRNA expression was significant only in lung adenocarcinoma. As shown in Figure 3D, $C 1 Q A, C 1 Q B$, and $C 1 Q C$ mRNA expression levels negatively correlated with an OS rate of the patients with lung adenocarcinoma $(p<0.05)$; no correlation with OS was observed in squamous cell carcinoma (Table 1).

IHC in lung adenocarcinoma revealed $\mathrm{Clq}$ staining in the stroma and some macrophage-like positive cells into the tumor mass (Figure 2C).

\section{DISCUSSION}

In this paper, we performed bioinformatics analysis to explore if $\mathrm{C} 1 \mathrm{q}$ level could act as a possible prognostic marker in various carcinomas, in view of its reported dichotomous effects on cancer cells (pro- and anti-tumorigenic). C1q is present in colon, lung, breast, pancreatic carcinoma, and melanoma. $\mathrm{C} 1 \mathrm{q}$ can promote adhesion, proliferation and migration of melanoma cells (17). We found $\mathrm{C} 1 \mathrm{q}$ in abundance in all histological variants (epithelioid, sarcomatoid, and biphasic) of asbestosinduced malignant pleural mesothelioma. C1q bound high and low molecular weight $\mathrm{HA}$ and acted as a tumor-promoting factor (18). In addition, C1q exerted a protective effect against apoptosis, suggesting an overall pro-tumorigenic activity (17). However, Hong et al. recently observed that C1q, expressed in normal prostate, was downregulated in benign prostatic hyperplasia and prostate cancer (15). C1q was able to induce apoptosis and growth suppression of human prostate DU145 

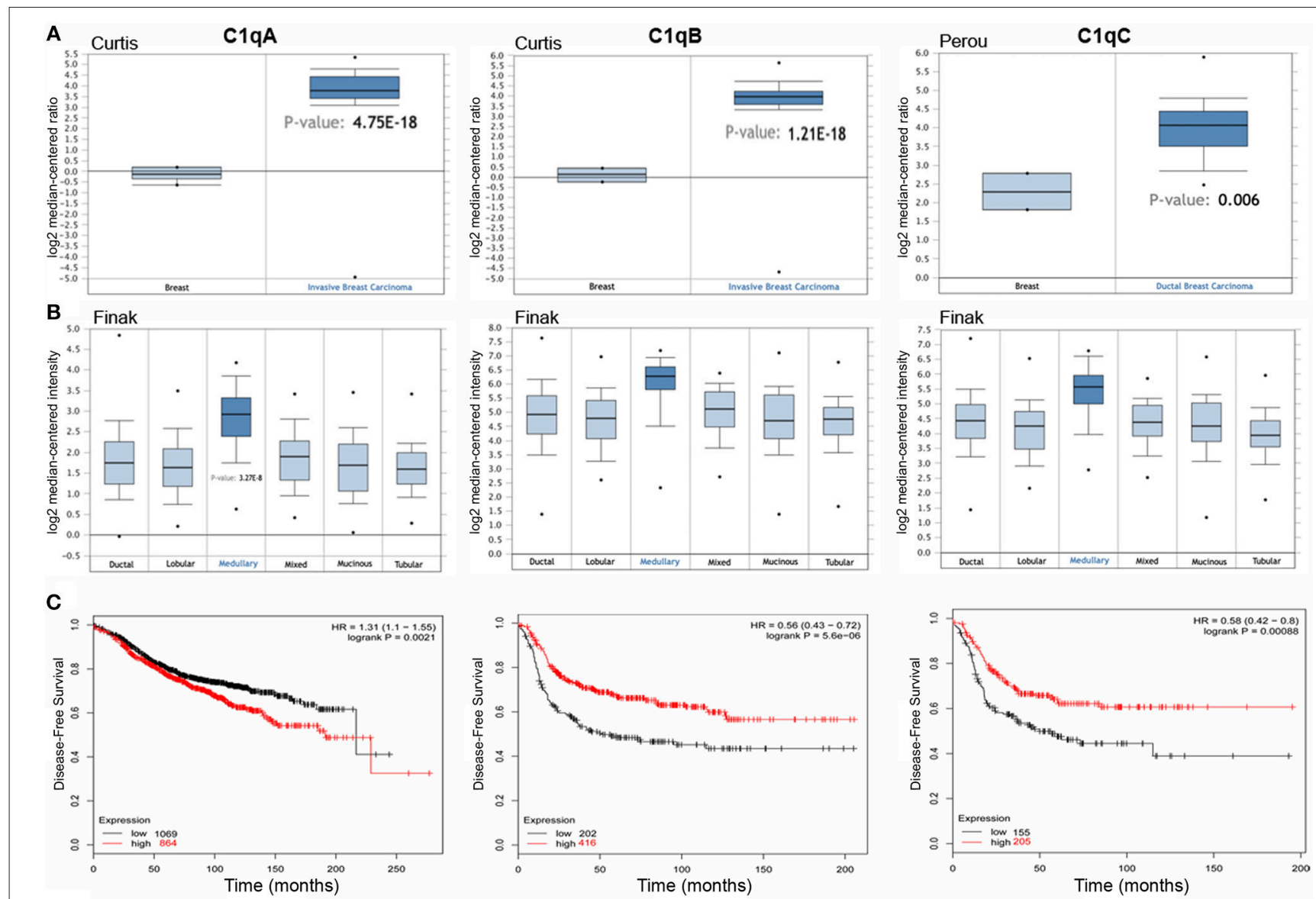

FIGURE 1 | C1QA, C1QB, and C1QC expression in invasive breast carcinoma. Curtis's datasets were used for bioinformatics analysis to explore C1QA and C1QB mRNAs expression in the breast cancer, whereas Perou's datasets was used for bioinformatics analysis to evaluate C1QC mRNA. A higher C1q mRNAs expression was detectable in invasive breast carcinoma compared to normal breast tissue (A). The analysis of the different breast carcinoma histotypes by Finak's dataset revealed how the medullary breast cancer presented the major intensity of C1q mRNA expression (B). According to the data Kaplan-Meir plotter, C1QA, C1QB, and C1QC mRNA expressions was positively linked to a disease-free survival (DFS) rate in patients with basal-like cancers (C) $(p<0.05)$ and to an overall survival (OS) rate with HER-2 positive cancers (Supplementary Table 2). HR, hazard ratio.

cells, through direct activation of the tumor suppressor WWdomain containing oxidoreductase (WOX1). C1q also have a pro-apoptotic effect on an ovarian cell line, SKOV3, acting via a TNF- $\alpha$ induced apoptosis pathway that involves upregulation of Bax and Fas (16).

In a syngeneic murine model of melanoma in C57BL/6 strain, C1q-deficient mice showed prolonged survival and slower tumor growth, as compared to wild-type mice (17). However, Bandini et al. found that neuT mice, a genetically engineered mouse model for mammary carcinoma that was made deficient for the ClqA chain (neuT-C1KO mice), manifested an accelerated tumor growth associated with an increased number of intratumoral vessels, compared to wild-type neuT mice. These differences in tumor progression were attributed to a reduced activation of WW domain containing oxidoreductase (WWOX) in C1q-deficient mice (25).

In view of these rather contradicting roles of $\mathrm{Clq}$ in tumor progression, we performed a systematic bioinformatics analysis of the expression of $\mathrm{Clq}$, and its correlation with the survival rate in different carcinoma histotypes, using Oncomine and KaplanMeier plotter tools. We selected the carcinomas that showed all the three chains of human C1q statistically significant for the prognosis; in several cases, the prognosis was differentially linked to the C1q chains, or limited to one or two C1q chains. We often noticed the mRNA encoding for only one or two C1q chains, something that would impede synthesis of a functional $\mathrm{C} 1 \mathrm{q}$ molecule. Indeed, we have provided evidence in the past that the expression of $\mathrm{C} 1 \mathrm{qC}$ chain is essential for the production of functional C1q by the endothelial cells of the decidua (26). Moreover, mesothelioma cells are impaired in $\mathrm{Clq}$ A chain synthesis (18).

Our bioinformatics analysis highlighted that high levels of C1q have a favorable prognostic index in BLBCs for DFS and HER $2^{+}$breast cancer for OS, (Graphical Abstract) consistent with the in vivo studies by Bandini et al. using C1q-deficient mice (25). Inflammation is a major characteristic of these types of tumors. One possible explanation for the observed positive association between $\mathrm{Clq}$ expression and favorable 

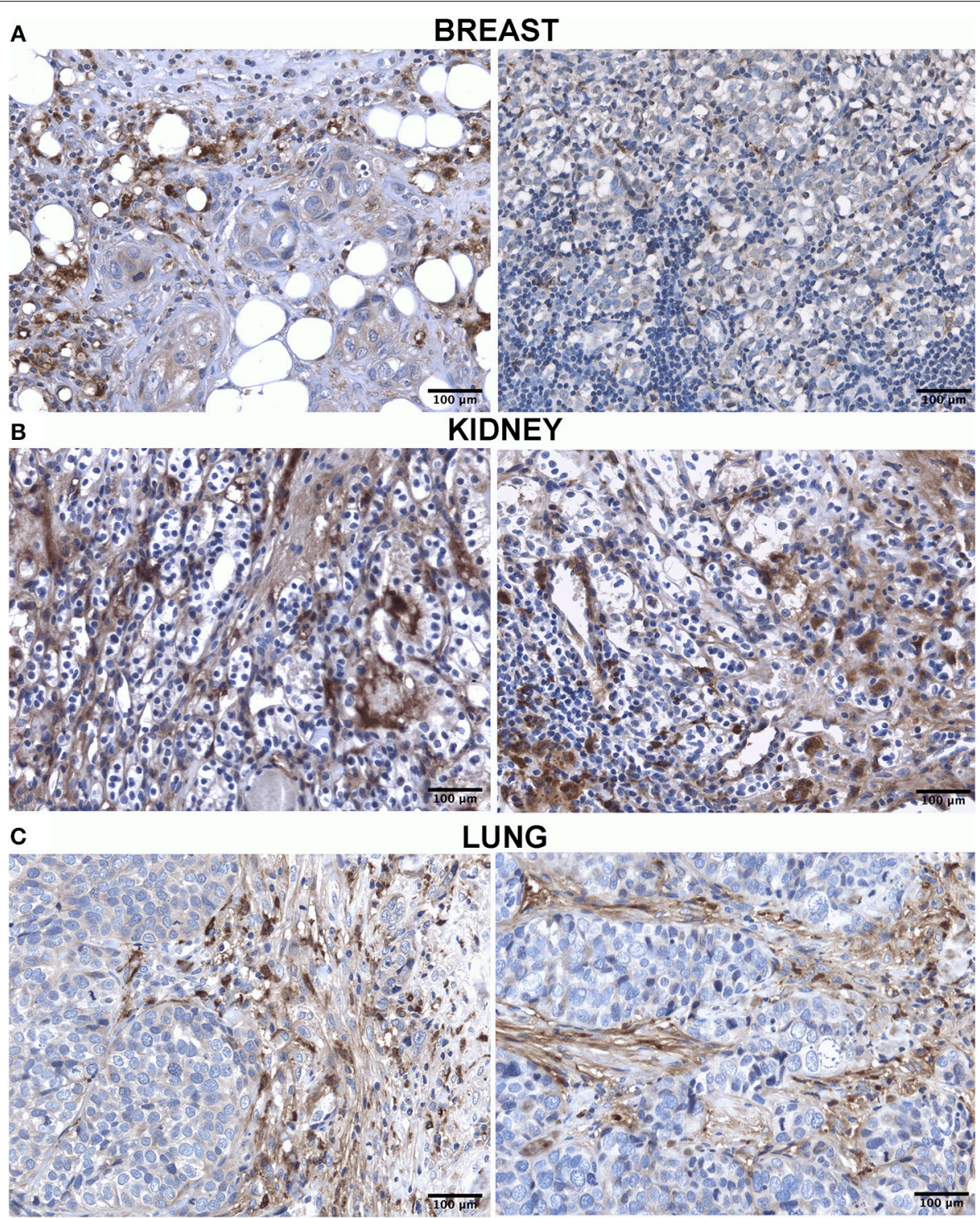

FIGURE 2 | Immunohistochemistry analysis for C1q in breast (A), kidney (B) and lung carcinoma (C). Representative microphotographs showing expression of C1q in different carcinoma. The expression of $\mathrm{C} 1 \mathrm{q}$ in carcinoma was observed in all tissues with differential distribution in the TME, as described in the result section. DAB (brown) chromogen was used to visualize the binding of anti-human C1q antibodies; scale bars, 50 $\mu \mathrm{m}$.

prognostic index could be due to the correlation between the presence of $\mathrm{Clq}$ and dendritic cells (CD11c positive cells) in TME. High CD11c expression in BLBCs is associated with a significantly higher OS $(p=0.047)$ as compared to low CD11c expression (27). Dendritic cells themselves can be a potential source of C1q within the TME $(28,29)$. C1q, although present, is not able to bind BLBC cells (MDA-MB-231), and hence, not able to promote tumor progression (unpublished data), probably due to downregulation of putative $\mathrm{C} 1 \mathrm{q}$ receptor(s). It is thus crucial to understand the differences in good prognosis survival between BLBCs and HER2 ${ }^{+}$breast cancer, the role of inflammation, and that of $\mathrm{Clq}$ in determining such differences.
Wilson et al. (30) found that C1q chain genes were enriched in the stroma compartment of triple-negative breast cancers. The analysis of publicly available data sets revealed that the genes encoding for the $\mathrm{C} 1 \mathrm{q}$ chains were associated with a poor prognosis in BLBC using the TCGA dataset (504 patients). In our analysis, using the GEO dataset that include 5,143 patients, we observed a positive prognostic effect for BLBCs in DFS and HER2-positive breast cancers in OS. The opposite results were obtained for CCRCCs and lung adenocarcinomas in OS.

A negative prognostic effect arose from the analysis of kidney and lung carcinomas (Graphical Abstract). The most frequent histological subtypes include CCRCC and PRCC (CCRCC $\sim 75 \%$; PRCC $\sim 10 \%$ ) (31). The expression of C1q in kidney 


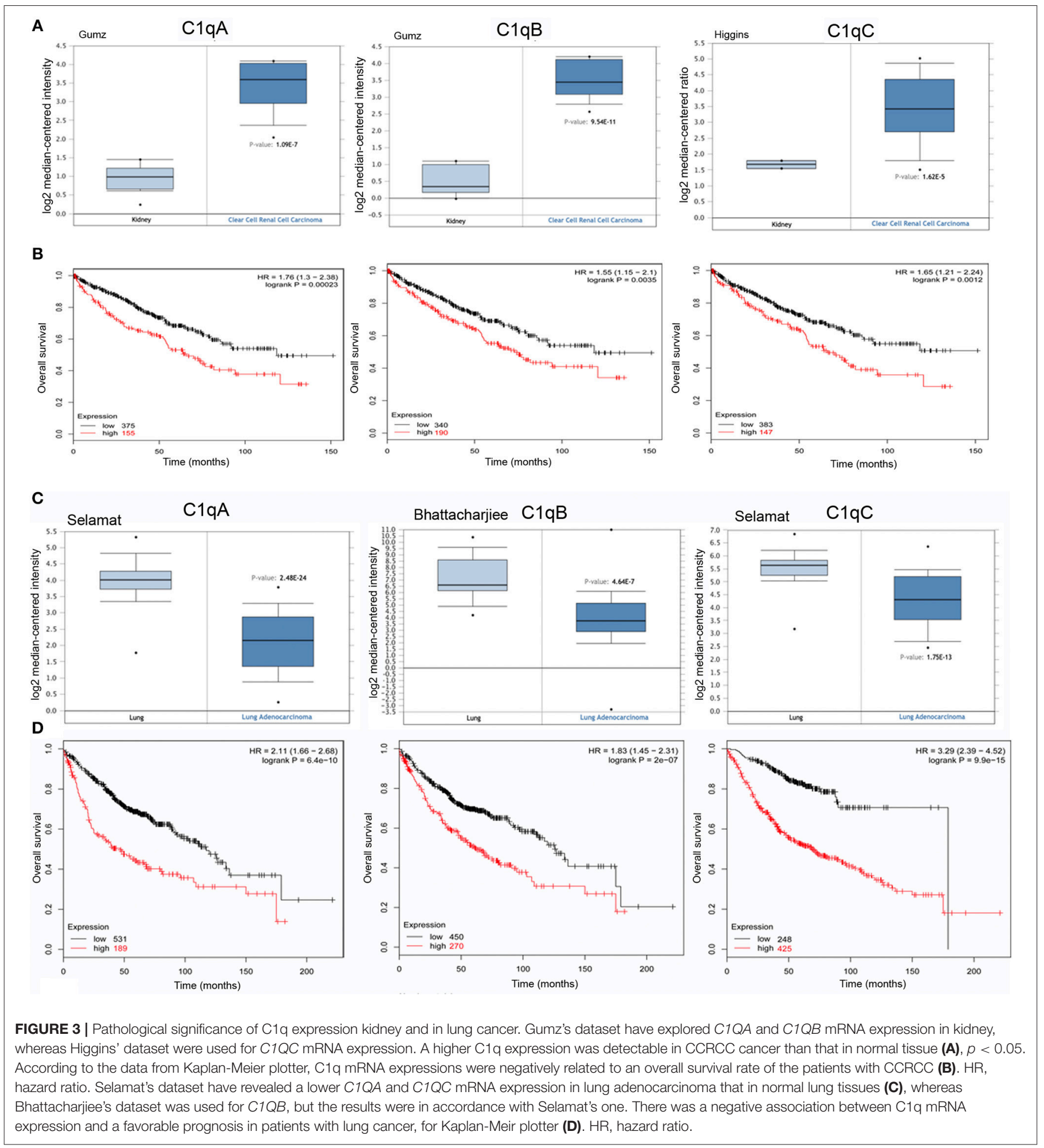

cancer is increased as compared to normal kidney tissue (Figure 3A) and $\mathrm{C} 1 \mathrm{q}$ has a negative prognostic effect in the case of CCRCC (Figure 3B); no association was evident for PRCC. CCRCC tumor is characterized by an increased response to HIF that promotes blood vessel growth. Targeted therapies directed against VEGF, VEGF receptor, and mTOR play a crucial role in the management of metastatic CCRCC (32). We can hypothesize that $\mathrm{Clq}$ can also participate in promoting angiogenic processes in this particular tumor (14).

C1q has a negative prognostic value in lung tumors limited to adenocarcinomas, the most common form of lung cancer (Figure 3D). According to the WHO classification of lung 
tumors, there are four major histological types: adenocarcinomas, squamous cell carcinomas, large cell carcinomas, and small cell carcinomas (33). It is worth noting that $\mathrm{C} 1 \mathrm{q}$ expression is reduced in lung cancer compared to the normal lung as we observed for surfactant protein D (SP-D) (34). Although C1q expression in lung cancer is lower than in normal tissue, lung cancer cells bind $\mathrm{C1q}$ present in the tumor microenvironment and activate the classical complement pathway (35). Tumor transformation is also concomitant with the loss of key defense molecules entrusted with early recognition and removal of the altered self (36).

A number of factors can modulate the role of $\mathrm{Clq}$ in the TME. C1q interaction with the ECM components can adversely interrupt its putative functions, as is the case with HA. It is also possible that certain tumors downregulate the putative receptor for $\mathrm{Clq}$ in order to escape possible apoptosis induction. Proliferative and apoptotic responses to $\mathrm{C} 1 \mathrm{q}$ can be dictated by distinct receptors that are yet to be discovered. Last but not the least, the orientation of the $\mathrm{C} 1 \mathrm{q}$ molecule, while engaging with the tumor cells, can also define the C1q-mediated implications. Our study encompasses all the above-mentioned possibilities, including tumor heterogeneity.

\section{ETHICS STATEMENT}

This study was carried out as per the recommendations of governmental guidelines, and approved by the CEUR (Comitato Etico Unico Regionale, FVG, Italy; number 34/2016). All subjects gave written informed consent in accordance with the Declaration of Helsinki.

\section{REFERENCES}

1. Lu J, Kishore U. C1 complex: an adaptable proteolytic module for complement and non-complement functions. Front Immunol. (2017) 8:592. doi: 10.3389/fimmu.2017.00592

2. Kishore U, Gaboriaud C, Waters P, Shrive AK, Greenhough TJ, Reid KB, et al. $\mathrm{C} 1 \mathrm{q}$ and tumor necrosis factor superfamily: modularity and versatility. Trends Immunol. (2004) 25:551-61. doi: 10.1016/j.it.2004.08.006

3. Kishore U, Ghai R, Greenhough TJ, Shrive AK, Bonifati DM, Gadjeva MG, et al. Structural and functional anatomy of the globular domain of complement protein C1q. Immunol Lett. (2004) 95:113-28. doi: 10.1016/j.imlet.2004.06.015

4. Thielens NM, Tedesco F, Bohlson SS, Gaboriaud C, Tenner AJ. C1q: A fresh look upon an old molecule. Mol Immunol. (2017) 89:73-83. doi: 10.1016/j.molimm.2017.05.025

5. Petry F, Botto M, Holtappels R, Walport MJ, Loos M. Reconstitution of the complement function in C1q-deficient $\left(\mathrm{Clqa}^{-/-}\right)$mice with wild-type bone marrow cells. J Immunol. (2001) 167:4033-7. doi: 10.4049/jimmunol.167.7.4033

6. Nayak A, Pednekar L, Reid KB, Kishore U. Complement and noncomplement activating functions of $\mathrm{Clq}$ : a prototypical innate immune molecule. Innate Immun. (2012) 18:350-63. doi: 10.1177/1753425910396252

7. Sellar GC, Cockburn D, Reid KB. Localization of the gene cluster encoding the $\mathrm{A}, \mathrm{B}$, and $\mathrm{C}$ chains of human $\mathrm{C} 1 \mathrm{q}$ to $1 \mathrm{p} 34.1-1 \mathrm{p} 36.3$. Immunogenetics. (1992) 35:214-6.

8. Ghebrehiwet B, Kandov E, Kishore U, Peerschke EIB. Is the A-chain the engine that drives the diversity of $\mathrm{Clq}$ functions? revisiting its unique structure. Front Immunol. (2018) 9:162. doi: 10.3389/fimmu.2018. 00162

\section{AUTHOR CONTRIBUTIONS}

$\mathrm{AM}$ and $\mathrm{RB}$ : conception and design. $\mathrm{CA}, \mathrm{DB}$, and $\mathrm{AR}$ : development of methodology. BB, DB, CA, and GZ: acquisition of data. AM, DB, BB, FZ and CA: analysis and interpretation of data (e.g., statistical analysis, biostatistics, and computational analysis). RB, UK, CA, PZ, and GR: writing, review, and/or revision of the manuscript. RB: study supervision.

\section{FUNDING}

This work was supported by grants from the Institute for Maternal and Child Health, IRCCS Burlo Garofolo, Trieste, Italy (RC 20/16, RC 23/18), AIRC to Claudio Tripodo. Fondazione Cassa di Risparmio Trieste to RB.

\section{ACKNOWLEDGMENTS}

We thank Andrea Balduit, Fleur Bossi (IRCCS, Burlo Garofolo, Trieste, Italy) and Alessandro Gulino (Department of Human Pathology, University of Palermo) for the immunohistochemical analysis. The contribution of Nicolò Morosini for the C1 visualization in Graphical Abstract is acknowledged.

\section{SUPPLEMENTARY MATERIAL}

The Supplementary Material for this article can be found online at: https://www.frontiersin.org/articles/10.3389/fimmu. 2019.00865/full\#supplementary-material

9. Reid KBM. Complement component C1q: historical perspective of a functionally versatile, and structurally unusual, serum protein. Front Immunol. (2018) 9:764. doi: 10.3389/fimmu.2018.00764

10. Agostinis C, Bulla R, Tripodo C, Gismondi A, Stabile H, Bossi F, et al. An alternative role of $\mathrm{Clq}$ in cell migration and tissue remodeling: contribution to trophoblast invasion and placental development. J Immunol. (2010) 185:44209. doi: 10.4049/jimmunol.0903215

11. Agostinis C, Tedesco F, Bulla R. Alternative functions of the complement protein C1q at embryo implantation site. J Reprod Immunol. (2017) 119:7480. doi: 10.1016/j.jri.2016.09.001

12. Singh J, Ahmed A, Girardi G. Role of complement component C1q in the onset of preeclampsia in mice. Hypertension. (2011) 58:716-24. doi: 10.1161/HYPERTENSIONAHA.111.175919

13. Agostinis C, Stampalija T, Tannetta D, Loganes C, Vecchi Brumatti L, De Seta F, et al. Complement component C1q as potential diagnostic but not predictive marker of preeclampsia. Am J Reprod Immunol. (2016) 76:475-81. doi: 10.1111/aji.12586

14. Bossi F, Tripodo C, Rizzi L, Bulla R, Agostinis C, Guarnotta C, et al. C1q as a unique player in angiogenesis with therapeutic implication in wound healing. Proc Natl Acad Sci USA. (2014) 111:4209-14. doi: 10.1073/pnas.1311968111

15. Hong Q, Sze CI, Lin SR, Lee MH, He RY, Schultz L, et al. Complement C1q activates tumor suppressor WWOX to induce apoptosis in prostate cancer cells. PLoS ONE. (2009) 4:e5755. doi: 10.1371/journal.pone.0005755

16. Kaur A, Sultan SH, Murugaiah V, Pathan AA, Alhamlan FS, Karteris $\mathrm{E}$, et al. Human $\mathrm{C} 1 \mathrm{q}$ induces apoptosis in an ovarian cancer cell line via tumor necrosis factor pathway. Front Immunol. (2016) 7:599. doi: 10.3389/fimmu.2016.00599

17. Bulla R, Tripodo C, Rami D, Ling GS, Agostinis C, Guarnotta C, et al. $\mathrm{Clq}$ acts in the tumour microenvironment as a cancer-promoting factor 
independently of complement activation. Nat Commun. (2016) 7:10346. doi: 10.1038/ncomms10346

18. Agostinis C, Vidergar R, Belmonte B, Mangogna A, Amadio L, Geri P, et al. Complement protein $\mathrm{C} 1 \mathrm{q}$ binds to hyaluronic acid in the malignant pleural mesothelioma microenvironment and promotes tumor growth. Front Immunol. (2017) 8:1559. doi: 10.3389/fimmu.2017.01559

19. Markiewski MM, DeAngelis RA, Benencia F, Ricklin-Lichtsteiner SK, Koutoulaki A, Gerard C, et al. Modulation of the antitumor immune response by complement. Nat Immunol. (2008) 9:1225-35. doi: 10.1038/ ni. 1655

20. Corrales L, Ajona D, Rafail S, Lasarte JJ, Riezu-Boj JI, Lambris JD, et al. Anaphylatoxin C5a creates a favorable microenvironment for lung cancer progression. J Immunol. (2012) 189:4674-83. doi: 10.4049/jimmunol. 1201654

21. Riihila P, Nissinen L, Farshchian M, Kallajoki M, Kivisaari A, Meri S, et al. Complement component C3 and complement factor B promote growth of cutaneous squamous cell carcinoma. Am J Pathol. (2017) 187:1186-97. doi: 10.1016/j.ajpath.2017.01.006

22. Rhodes DR, Yu J, Shanker K, Deshpande N, Varambally R, Ghosh D, et al. ONCOMINE: a cancer microarray database and integrated data-mining platform. Neoplasia. (2004) 6:1-6. doi: 10.1016/S1476-5586(04)80047-2

23. Rhodes DR, Kalyana-Sundaram S, Mahavisno V, Varambally R, Yu J, Briggs $\mathrm{BB}$, et al. Oncomine 3.0: genes, pathways, and networks in a collection of 18,000 cancer gene expression profiles. Neoplasia. (2007) 9:166-80. doi: $10.1593 /$ neo. 07112

24. Lanczky A, Nagy A, Bottai G, Munkacsy G, Szabo A, Santarpia L, et al. miRpower: a web-tool to validate survival-associated miRNAs utilizing expression data from 2178 breast cancer patients. Breast Cancer Res Treat. (2016) 160:439-46. doi: 10.1007/s10549-016-4013-7

25. Bandini S, Macagno M, Hysi A, Lanzardo S, Conti L, Bello A, et al. The noninflammatory role of $\mathrm{C} 1 \mathrm{q}$ during Her2/neu-driven mammary carcinogenesis. Oncoimmunology. (2016) 5:e1253653. doi: 10.1080/2162402X.2016. 1253653

26. Bulla R, Agostinis C, Bossi F, Rizzi L, Debeus A, Tripodo C, et al. Decidual endothelial cells express surface-bound $\mathrm{Clq}$ as a molecular bridge between endovascular trophoblast and decidual endothelium. Mol Immunol. (2008) 45:2629-40. doi: 10.1016/j.molimm.2007.12.025

27. Lee H, Lee HJ, Song IH, Bang WS, Heo SH, Gong G, et al. CD11c-positive dendritic cells in triple-negative breast cancer. in vivo. (2018) 32:1561-9. doi: 10.21873/invivo.11415

28. Castellano G, Trouw LA, Fiore N, Daha MR, Schena FP, van Kooten C. Infiltrating dendritic cells contribute to local synthesis of $\mathrm{C} 1 \mathrm{q}$ in murine and human lupus nephritis. Mol Immunol. (2010) 47:2129-37. doi: 10.1016/j.molimm.2010.02.006

29. Castellano G, Woltman AM, Nauta AJ, Roos A, Trouw LA, Seelen MA, et al. Maturation of dendritic cells abrogates C1q production in vivo and in vitro. Blood. (2004) 103:3813-20. doi: 10.1182/blood-2003-09-3046

30. Winslow S, Leandersson K, Edsjo A, Larsson C. Prognostic stromal gene signatures in breast cancer. Breast Cancer Res. (2015) 17:23. doi: 10.1186/s13058-015-0530-2

31. Lopez-Beltran A, Carrasco JC, Cheng L, Scarpelli M, Kirkali Z, Montironi R. 2009 update on the classification of renal epithelial tumors in adults. Int J Urol. (2009) 16:432-43. doi: 10.1111/j.1442-2042.2009.02302.x

32. Su D, Singer EA, Srinivasan R. Molecular pathways in renal cell carcinoma: recent advances in genetics and molecular biology. Curr Opin Oncol. (2015) 27:217-23. doi: 10.1097/CCO.0000000000000186

33. Travis WD, Brambilla E, Burke AP, Marx A, Nicholson AG. Introduction to The 2015 World Health Organization Classification of Tumors of the Lung, Pleura, Thymus, and Heart. J Thorac Oncol. (2015) 10:1240-2. doi: $10.1097 /$ JTO.0000000000000663

34. Mangogna A, Belmonte B, Agostinis C, Ricci G, Gulino A, Ferrara I, et al. Pathological significance and prognostic value of surfactant protein D in cancer. Front Immunol. (2018) 9:1748. doi: 10.3389/fimmu.2018.01748

35. Ajona D, Pajares MJ, Corrales L, Perez-Gracia JL, Agorreta J, Lozano MD, et al. Investigation of complement activation product c4d as a diagnostic and prognostic biomarker for lung cancer. J Natl Cancer Inst. (2013) 105:1385-93. doi: 10.1093/jnci/djt205

36. Vandivier RW, Ogden CA, Fadok VA, Hoffmann PR, Brown KK, Botto M, et al. Role of surfactant proteins $\mathrm{A}, \mathrm{D}$, and $\mathrm{Clq}$ in the clearance of apoptotic cells in vivo and in vitro: calreticulin and CD91 as a common collectin receptor complex. J Immunol. (2002) 169:3978-86. doi: 10.4049/jimmunol.169. 7.3978

Conflict of Interest Statement: The authors declare that the research was conducted in the absence of any commercial or financial relationships that could be construed as a potential conflict of interest.

Copyright (๑) 2019 Mangogna, Agostinis, Bonazza, Belmonte, Zacchi, Zito, Romano, Zanconati, Ricci, Kishore and Bulla. This is an open-access article distributed under the terms of the Creative Commons Attribution License (CC BY). The use, distribution or reproduction in other forums is permitted, provided the original author(s) and the copyright owner(s) are credited and that the original publication in this journal is cited, in accordance with accepted academic practice. No use, distribution or reproduction is permitted which does not comply with these terms. 